\title{
End-to-end Vector Dynamics of Non-entangled Polymers in Lamellar Block Copolymer Melts: the Role of Junction Point Motion
}

Reidar Lund, ${ }^{1}$ Fabienne Barroso-Bujans, ${ }^{2}$ Mohammed Zakaria Slimani, ${ }^{2}$ Angel J. Moreno, ${ }^{3, *}$ Lutz Willner, ${ }^{4}$ Dieter Richter, ${ }^{4}$ Angel Alegría,,${ }^{3,5}$ and Juan Colmenero ${ }^{2,3,5}$

${ }^{1}$ Department of Chemistry, University of Oslo, Postboks 1033 Blindern, 0315 Oslo, Norway

${ }^{2}$ Donostia International Physics Center, Paseo Manuel de Lardizabal 4, 20018 San Sebastián, Spain.

${ }^{3}$ Centro de Física de Materiales (CSIC, UPV/EHU) and Materials Physics Center MPC, Paseo Manuel de Lardizabal 5, 20018 San Sebastián, Spain.

${ }_{4}^{4}$ Juelich Centre for Neutron Science (JCNS) and Institute for Complex Systems (ICS), Forschungszentrum Juelich GmbH, 52425 Juelich, Germany ${ }^{5}$ Departamento de Física de Materiales, Universidad del País Vasco (UPV/EHU), Apartado 1072, 20080 San Sebastián, Spain.

By using dielectric spectroscopy, we investigate the chain dynamics of nonentangled polyisoprene (PI) under soft confinement in lamellar domains of block copolymer melts with polydimethylsiloxane (PDMS). The data show a dramatic difference in the end-to-end vector dynamics of the PI-blocks as compared not only with that of the corresponding homopolymer PI-chains, but also with respect to previous results for the same blocks under soft confinement in cylindrical domains. Two contributions to the dielectric normal mode relaxation are detected. The data are analyzed by means of a model including contributions from internal chain modes (accounting for the fastest component), and a slow component attributed to the 
junction point dynamics. The contribution of the internal chain modes is modelled according to the analysis of the Rouse modes obtained from simulations of a generic bead-spring model for strongly segregated symmetric diblock copolymers. In this way it is shown that the internal chain modes of the blocks have time scales close to those expected from the homopolymer chain independently on the structural details. In contrast, the contribution attributed to the junction point dynamics depends critically on minor structural differences. We interpret these findings as a result of the presence of fast moving defects and/or grain boundaries in the lamellar structures formed by these relatively short, non-entangled diblock copolymers.

*Corresponding author: wabmosea@ehu.es 


\section{INTRODUCTION}

As a consequence of the ever-lasting pursuit of manipulating material properties on a smaller and smaller scale, a strong effort has been dedicated to understand the physics controlling the properties at the nanoscale. Here polymer materials and especially block copolymers play a very important role for many technological applications, where these materials can be used for litography, nano-templates, membranes etc. [1-4]. A particular attractive feature of block copolymer systems is the easy control of their length scales and the morphology that can be obtained by varying the molecular weight, block composition, chemistry of the blocks, temperature, etc. In fact even simple A-B block copolymer melts are known to self-assemble into a wide range of structures ranging from locally planar lamellae to highly curved structures such as cylindrical and spherical phases, as well as bicontinuous network phases such as the gyroid phase [1].

While the thermodynamics and structure of these systems have been extensively studied over the last decades [2,5-7], less attention has been paid to their dynamic properties. Studies on the dynamics of block copolymers have mostly focused on rheological or diffusional properties [8-13]. In a series of works (see review in Ref. [13]), the self-diffusion of poly(ethylene-alt-propylene)-poly(dimethylsiloxane) (PEP-PDMS) block copolymers forming different morphologies was studied by using NMR. These studies showed that the largescale motion of the chains is anisotropic, following an activated diffusion across the phase boundaries (interdomain diffusion). This anisotropic motion was detected directly by forced Rayleigh scattering on aligned lamellar samples by Lodge and coworkers [9]. Much less work has been dedicated to characterize the fast chain dynamic processes, such as the segmental $\alpha$-relaxation or the global end-to-end vector (normal mode, NM) relaxation. Both processes can be studied by broadband dielectric spectroscopy [14]. Previous investigations have shown that the segmental $\alpha$-relaxation can be either slowed down $[15,16]$ or accelerated if the component is confined by a slower or faster phase respectively $[15,17]$. Recent simulations confirmed this picture $[18,19]$. A recent study by Sanz et al. [16] addressed the effect on the $\alpha$-relaxation when the confining matrix crosses its glass transition temperature, $T_{g}$, i.e., when going from a 'hard' to a 'soft' confinement. It was found that the low- $T_{\mathrm{g}}$ (fast) component did not exhibit any discontinuity upon crossing the $T_{\mathrm{g}}$ of the confining (slow) phase. However, they observed a dynamic crossover by increasing temperature and crossing 
the order-disorder transition.

Concerning the global chain dynamics most studies were done on poly(isoprene)poly(styrene) (PI-PS) block copolymers, with PI forming the minority phase [15, 20]. Very recently, several works have addressed in more detail the chain dynamics of the confined phase by studying the NM of PI in other diblock and triblock copolymers with PS [21-23]. In general, it was found that both the $\alpha$ - and NM-process of the PI-block were considerably slowed down with respect to the PI-homopolymer. This effect was attributed to the grafting of the PI-chains to the outer block phase of PS. However, in a recent study on cylindrical and spherical phases of PI-PDMS copolymers, where the minority PI was confined by fluid PDMS, the opposite effect - an acceleration of the normal mode dynamics — was observed [24]. Similar results were found in neutron spin-echo experiments on the same [25] and other systems [26]. In summary, grafting of the blocks to the interface and confinement do not necessarily lead to a NM relaxation slower than in the corresponding homopolymers.

In this work we investigate by means of dielectric spectroscopy, the block end-to-end vector dynamics of non-entangled PI segregated in lamellar phases of diblock copolymers with PDMS. The experimental results are compared with simulations of a generic beadspring model for strongly segregated lamellae. We find that the end-to-end vector relaxation of the PI-blocks occurs in two steps. The first one is determined by the block internal Rouse modes, while the second one is controlled by the dynamics of the junction points. This later process results to be critically affected by minor structural details. This finding is tentatively interpreted as a consequence of the defects and/or phase boundaries motions associated to the relatively short blocks forming the lamellar structure. The article is organized as follows. In Section 2 we give experimental and simulation details. In Section 3 we present experimental results for structure and dynamics. In Sections 4 and 5 we analyze and discuss features of the Rouse modes by comparing experimental and simulation results. Conclusions are given in Section 6. 


\begin{tabular}{lcccc}
\hline & $M_{n}^{\mathrm{PI} a}(\mathrm{~kg} / \mathrm{mol})$ & $M_{n}^{\mathrm{PDMS} a}(\mathrm{~kg} / \mathrm{mol})$ & $f_{\mathrm{PI}}^{b)}$ & $10^{2} f_{\mathrm{PI}}^{-1} \Delta \varepsilon(273$ \\
& & & & \\
PI4 & 4.3 & - & 1 & $8.6 \pm 0.2$ \\
PI4-PDMS3.5 & 4.3 & 3.5 & 0.58 & $8.5 \pm 0.3$ \\
PI4-PDMS4 & 4.3 & 4.1 & 0.54 & $8.2 \pm 0.3$
\end{tabular}

TABLE I: Characteristic parameters of the PI-homopolymer and the PI-PDMS block copolymers studied in this work. ${ }^{a)}$ Molecular weight. The polydispersity is about 1.02-1.04. ${ }^{b)}$ Volume fraction of PI, based on the densities $d_{\mathrm{PI}}=0.91 \mathrm{~g} / \mathrm{cm}^{3}$ and $d_{\mathrm{PDMS}}=0.97 \mathrm{~g} / \mathrm{cm}^{3}$ respectively [25]. $\Delta \varepsilon$ is the dielectric strength of the normal mode (see below).

\section{EXPERIMENTAL AND SIMULATION DETAILS}

\section{A. Synthesis of PI and PI-PDMS block copolymers}

PI-PDMS block copolymers were synthesized by anionic living polymerization as described in the Supplementary Information of Ref. [24]. Initially, living PI solution (PILi) was prepared by the reaction of $\mathrm{t}-\mathrm{BuLi}$ and isoprene in benzene at room temperature for ca. $40 \mathrm{~h}$. One part of the resulting living PILi was terminated with thoroughly degassed methanol to produce PI. The PI homopolymer was used as reference material. The residual living PILi solution was used as macroinitiator for the preparation of the PI-PDMS block copolymers. The crossover to PDMS was accomplished by the reaction of living PILi with hexamethylcyclotrisiloxane $\left(\mathrm{D}_{3}\right)$ for $20 \mathrm{~h}$ in pure benzene at room temperature. Afterwards, the polymerization was allowed to proceed by the addition of THF as promoter. The reaction was first left for $4 \mathrm{~h}$ at room temperature and then cooled to $-20{ }^{\circ} \mathrm{C}$ for 7 days in order to reach high conversion and to suppress undesired side reactions. The polymerizations were terminated by the addition of chlorotrimethylsilane. The copolymers were subsequently precipitated in methanol and dried at $30^{\circ} \mathrm{C}$ in a vacuum oven until constant weight was achieved. The molecular weight characteristics of the PI and PI-PDMS block copolymers are summarised in Table I. PI-blocks in both copolymers and chains in pure PI have identical mass, $M_{n}^{\mathrm{PI}}=4.3 \mathrm{~kg} / \mathrm{mol}$, somewhat below the entanglement mass $M_{\mathrm{e}}^{\mathrm{PI}} \sim 6$ $\mathrm{kg} / \mathrm{mol}[27]$. The PDMS-blocks are also non-entangled $\left(M_{\mathrm{e}}^{\text {PDMS }} \sim 12 \mathrm{~kg} / \mathrm{mol}\right)$ [27]. 


\section{B. Broadband dielectric spectroscopy (BDS)}

Measurements of the complex dielectric permittivity $\left(\varepsilon^{*}=\varepsilon^{\prime}-\mathrm{i} \varepsilon^{\prime \prime}\right)$ vs. frequency were performed in the range $10^{-1}-10^{6} \mathrm{~Hz}$, using a Novocontrol high-resolution dielectric analyzer (Alpha-N analyzer). For dielectric measurements, an upper electrode of $20 \mathrm{~mm}$ diameter was placed on the previously prepared film over a gold coated $20 \mathrm{~mm}$ diameter disc, and a separation of $100 \mu \mathrm{m}$ between both electrodes was maintained by using a cross shaped Teflon spacer of small area. The sample cell was set in a cryostat, and its temperature was controlled via a nitrogen gas jet stream coupled with the Novocontrol Quatro controller. The dielectric experiments were performed isothermally by cooling from room temperature in steps of $5 \mathrm{~K}$ down to the calorimetric glass transition of PI (ca. $200 \mathrm{~K}$ ). Equivalent experiments on heating confirmed the reproducibility of the obtained data.

\section{Molecular dynamics simulations}

We simulated a bead-spring chain model for diblock copolymers, of 'species' A and B, based on the original Kremer-Grest model [28]. Each chain contained $N=30$ monomers ('beads') of both species. The total chain length $(2 N)$ is of the order of the estimated entanglement length for bead-spring polymers [28]. The simulation box contained a total of 704 chains. Non-bonded interactions between two monomers at a distance $r$ were given by a Lennard-Jones potential,

$$
V_{\alpha \beta}^{\mathrm{LJ}}(r)=4 \epsilon_{\alpha \beta}\left[\left(\frac{\sigma}{r}\right)^{12}-\left(\frac{\sigma}{r}\right)^{6}+\frac{1}{4}\right] H\left(2^{1 / 6} \sigma-r\right),
$$

where $\alpha, \beta \in\{\mathrm{A}, \mathrm{B}\}, \sigma=1$ is the bead size, and $H$ is the Heaviside function. Bonded interactions were given by a FENE potential [28],

$$
V_{\alpha \beta}^{\mathrm{FENE}}(r)=-\epsilon_{\alpha \beta} K_{\mathrm{F}} R_{0}^{2} \ln \left[1-\left(\frac{r}{R_{0} \sigma}\right)^{2}\right],
$$

with $K_{\mathrm{F}}=15$ and $R_{0}=1.5$. We used energy scales $\epsilon_{\mathrm{AA}}=0.35$ and $\epsilon_{\mathrm{BB}}=1$, for the self-interactions. The cross-interaction was varied in the range $0.2 \leq \epsilon_{\mathrm{AB}} \leq 9$, exploring the whole range between the limits of isotropic disordered phases and strongly segregated lamellar phases. All the simulations were peformed at fixed temperature $T=0.6$ [29]. In the following, time and length units in the simulations can be qualitatively mapped to 
picoseconds and nanometers [28]. The procedure for equilibration of the lamellar structure in the ordered phases, and other simulation details, are described in Ref. [18].

\section{Small-angle X-ray Scattering (SAXS)}

The structure of the block copolymers was investigated by SAXS over an extended temperature range of about 173-313 K, by using a Rigaku diffractometer. The $4.5 \mathrm{~m}$ long instrument consists of a MM002+ micro focus source, a 2D-200X gas detector and a 3 pin hole collimation system. The samples were prepared using a sandwich cell with Kapton windows into a Linkam stage to control the temperature with a stability of about $\pm 0.2 \mathrm{~K}$.

\section{RESULTS}

\section{A. Morphology}

The SAXS experiments confirm the lamellar morphology for these nearly symmetric diblock copolymers, as expected from their relatively high interaction parameter $\chi(\approx 0.13$ at $298 \mathrm{~K}$ [30]). Representative SAXS patterns are shown in Figure 1. As can be seen in

the figure, the SAXS patterns at low temperatures exhibit a pronounced sharp diffraction peak, at wavevector $Q_{\max } \approx 0.0052 \mathrm{~nm}^{-1}$ and $Q_{\max } \approx 0.0050 \mathrm{~nm}^{-1}$ for PI4-PDMS3.5 and PI4-PDMS4 respectively. They also show secondary diffraction peaks at $2 Q_{\max }$ and $3 Q_{\max }$, as expected for a well developed lamellar structure. However, secondary reflections are only well resolved for both copolymers at low temperatures. In principle, the suppression of the secondary Bragg peaks at higher temperatures might indicate some degree of disorder as that coming from strong fluctuations of the domains formed by these rather small blocks. However we think this is not a relevant effect in the present case. First, there is a concomitant overall decrease of the diffracted intensity that indicates the reduction of contrast between the PDMS and PI phases as temperature increases. This reduction of contrast makes difficult by itself to resolve the secondary diffraction peaks from the instrumental background, independently of eventual changes in the structure. Second, we find that the width of the main diffraction peak does not increase with temperature. Third, there is a clear correlation, on the whole temperature range investigated, between the interlamellar spacing $\left(d \sim 2 \pi / Q_{\max }\right)$ and the interaction parameter $\chi$, following the power law $Q_{\max } \sim \chi^{-1 / 6} N^{-2 / 3}$ 

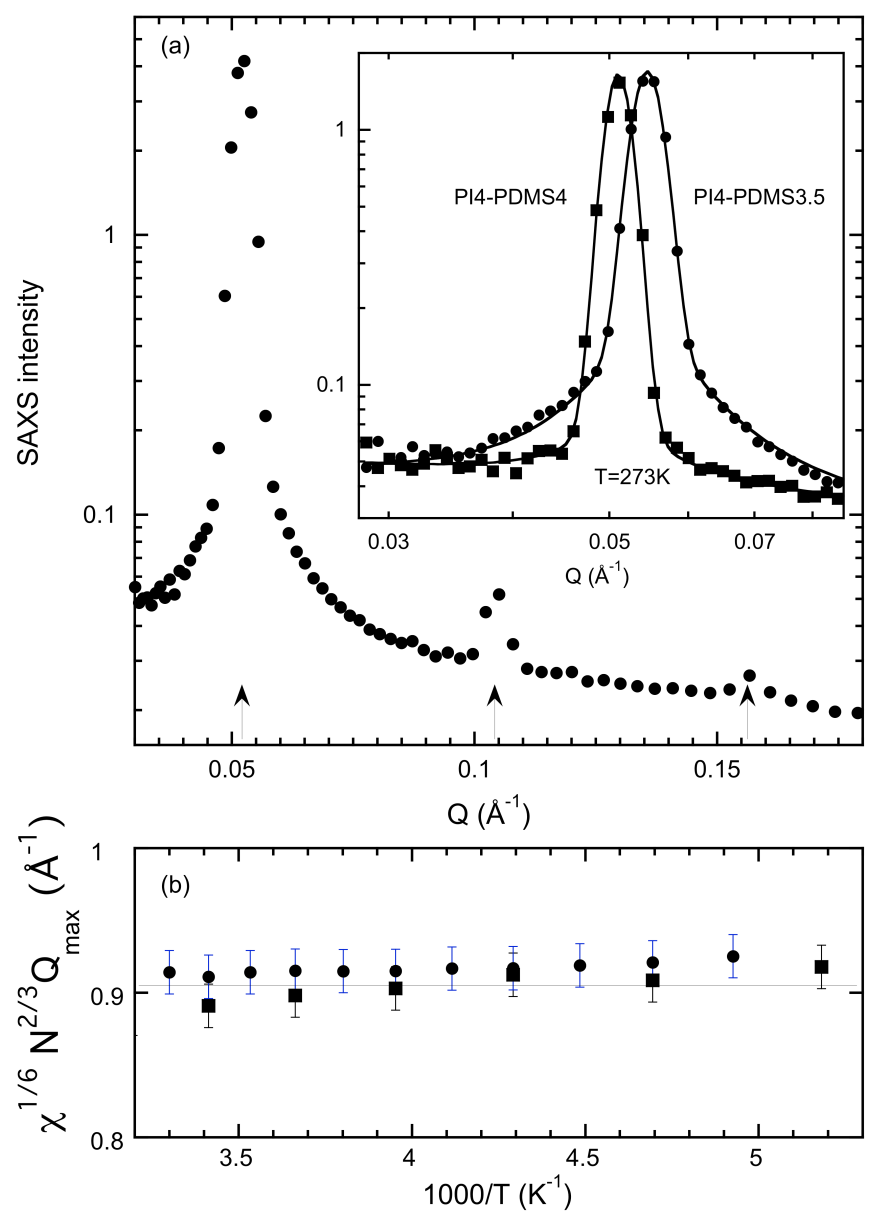

FIG. 1: Panel (a), main: representative small-angle X-ray scattering pattern collected at $173 \mathrm{~K}$ on the PI4-PDMS3.5 block copolymer. The arrows indicate the wavevectors for the main and secondary diffraction peaks. Panel (a), inset: detailed comparison between the main diffraction peaks of the two investigated copolymers at $273 \mathrm{~K}$. There, the solid lines are fits of the experimental peak to the sum of a Gaussian and a Lorentzian profile on top of the instrumental background. Panel (b): test of the theoretical result $Q_{\max } \sim \chi^{-1 / 6} N^{-2 / 3}$ predicted for the limit of strong segregation [31, 32]. Within error bars, the quantity $Q_{\max } \chi^{1 / 6} N^{2 / 3}$ is temperature independent (compare data with horizontal line), in agreement with theory. In all panels circles and squares are data for PI4-PDMS3.5 and PI4-PDMS4 respectively.

predicted by Semenov [31, 32] for the limit of strong segregation (see lower panel of Figure 1). All these observations point to a well developed lamellar structure in all the investigated cases.

At this point it must be mentioned that, as a direct consequence of the former power law, chains in strongly segregated lamellae do not adopt Gaussian conformations but are 
stretched. This might complicate the direct comparison of our experimental and simulation results with predictions of the Rouse model, which indeed assumes that chain conformations are Gaussian [33]. However, for the specific case of the short non-entangled chains investigated here, non-Gaussian effects only play a minor role. This can be argued as follows. For the most segregated lamellae in the simulations $\left(\epsilon_{\mathrm{AB}}=9\right)$, we can qualitatively estimate the $\chi$-parameter as of the order of $\chi \sim\left[\epsilon_{\mathrm{AB}}-\left(\epsilon_{\mathrm{AA}}+\epsilon_{\mathrm{BB}}\right) / 2\right]\left(k_{\mathrm{B}} T\right)^{-1}$. Thus, we find a value $\chi \sim 8$, much higher than the experimental one. By analyzing the end-to-end radii (not shown) of the simulated blocks and comparing them with the homopolymer counterparts, we find an stretching factor of just a $10 \%$ for the most segregated case. Therefore stretching of the experimental PI-blocks must be even weaker, since $\chi$ is much lower and both systems have comparable block lengths (somewhat below the entanglement length). In summary, chain stretching is not a relevant feature for the discussion of the end-to-end relaxation in our experimental and simulation results.

\section{B. Dielectric relaxation}

Dielectric relaxation allows to determine the dynamics of the dipolar entities in the sample. In particular, in the block copolymers here investigated both PDMS and PI present measurable dielectric relaxations. However they are detectable in a rather different temperature range. Whereas the dielectric relaxation of PDMS in the explored frequency range is detected at low temperatures $(T<170 \mathrm{~K})$, that of PI takes place mainly above the glass transition temperature of PI, i.e. at $T>200 \mathrm{~K}$. Thus, in the block copolymer where both phases are strongly segregated, the dielectric relaxation above $200 \mathrm{~K}$ will essentially reflect the dynamics of the PI-phase. Furthermore, since both phases present rather similar dielectric permittivities on the whole explored frequency range, no significant effects related with the local field differences are expected. This has been recently demonstrated for a similar system in Ref. [21]. Consequently, the measured dielectric losses of the PI-PDMS block polymers at temperatures above $200 \mathrm{~K}$ can be identified in a good approximation with the dielectric losses in the PI-phase, once the concentration of PI is taken into account.

BDS measurements on pure PI above the glass transition reveal two resolved dielectric loss peak contributions (see Figure 2). The first one, in the higher frequency range, is related to the segmental dynamics ( $\alpha$-relaxation), universally detected in polymer melts. The second 


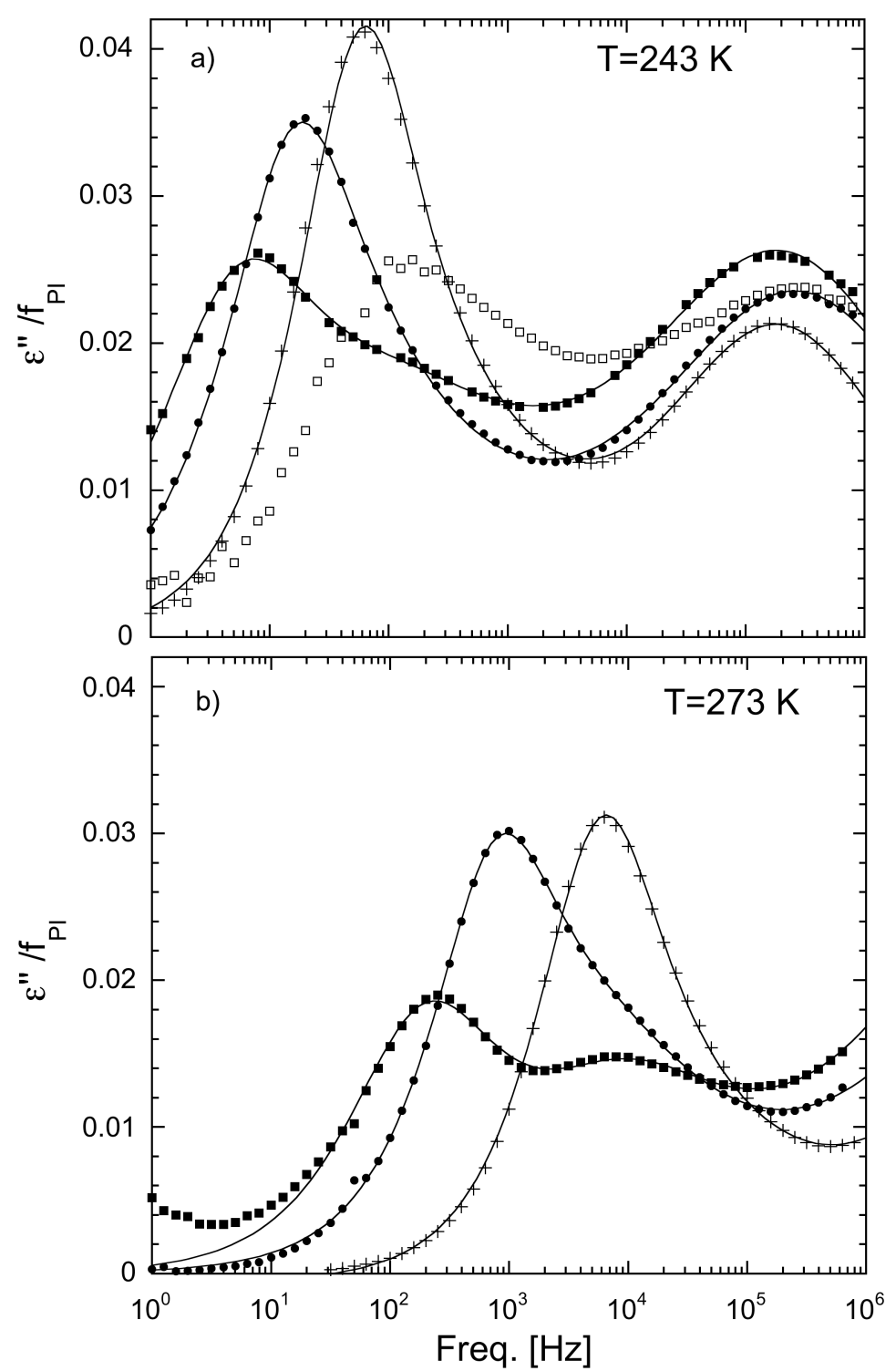

FIG. 2: Dielectric relaxation of the PI blocks in the different block copolymer mesophases: lamellar PI4PDMS3.5 (filled circles), lamellar PI4-PDMS4 (filled squares), cylindrical PI4-PDMS25 (empty squares [24]). Also included are data of pure PI4 (crosses). Panel a) shows the frequency dependence of the reduced loss permittivity for the three block copolymers at $243 \mathrm{~K}$, where both the NM and the $\alpha$-relaxation are clearly visible. In panel b) we show the NM relaxation of the PI blocks in the lamellar mesophase at a higher temperature $(273 \mathrm{~K})$, where the bimodal character of the NM becomes more evident. The bending-up in the data of PI4-PDMS4 at $\omega<10$ corresponds to the conductivity contribution, which is likely due to the higher content of PDMS in this sample. In both panels the data of pure PI at a temperature $5 \mathrm{~K}$ above have been included. By using this temperature shift, the loss peak of the $\alpha$-relaxation of pure PI takes place in a similar frequency range as in the copolymers. Lines correspond to data fitting as described in the main text. 
contribution, at lower frequencies, reflects the end-to-end vector fluctuations (normal mode relaxation). In BDS this feature is observed for the so-called type-A polymers, i.e. the polymers having a net dipole moment along the chain contour [14]. Representative BDS data collected on the lamellar copolymers and the corresponding pure PI are shown in Figure 2a, where the dielectric loss permittivity, $\varepsilon^{\prime \prime}$, is plotted as a function of frequency. To facilitate the comparison between data sets, for each sample the data have been normalized to the corresponding PI fraction. In the figure we also include published data of the same PI block in a highly asymmetric PI-PDMS diblock copolymer with cylindrical morphology [24]. This highlights the relevance of the specific morphology on the NM-relaxation. Indeed clear differences are found between the NM-peaks in the lamellar and cylindrical phases. It must be mentioned that the comparison with the PI homopolymer is not made at the same temperature but $5 \mathrm{~K}$ above. The reason for this choice is to compare results in all samples at a common value of the timescale of the respective segmental dynamics. This is demonstrated in Figure 2a by the $\alpha$-relaxation peak of PI observed for all samples at about $10^{5} \mathrm{~Hz}$. Therefore, in this way the effect of the presence in the copolymers of a much more flexible component (PDMS) seems to be somehow compensated. It is also noticeable that the $\alpha$-relaxation peak in the two lamellar copolymers is quite similar to that of the copolymer in the cylindrical morphology, which has a much higher PDMS fraction. This result evidences that the effects of the presence of the, much more flexible and faster, PDMS block on the $\alpha$-dynamics of the PI block is restricted to a few segments close to the junction point. This is consistent with recent computational investigations on the $\alpha$-relaxation in bead-spring copolymers with lamellar [18], cylindrical and spherical morphologies [19]. A thorough experimental characterization of the segmental dynamics in the different morphologies of the PI-PDMS copolymers is beyond the scope of this work and will be shown elsewhere [34].

The most striking result from the present BDS experiments is the bimodal character of the NM relaxation of the PI blocks in the PI-PDMS lamellae, unlike the single NM peak observed in the cylindrical and spherical morphologies [24]. This feature becomes more evident at higher temperatures, as shown in Figure 2b. An additional probe that the bimodal character of the NM relaxation is directly connected with the lamellar morphology is the fact that bimodality disappears suddenly above the order-disorder-transition (ODT) temperature (See Supp. Info.). The NM bimodal character in the lamellar phase is characterized by a well-defined main peak followed by a secondary peak or shoulder at higher frequencies. 
The main peak is located at lower frequencies than in pure PI whereas, noteworthy, the secondary component is located in the same frequency range of the NM peak of pure PI, once the temperature is properly corrected according to the shift of the $\alpha$-relaxation of pure PI. In Ref. [24] the single NM peak of the PI blocks segregated in cylindrical and spherical morphologies was observed in the same range than that of the corresponding pure PI [24]. This result is reproduced in Figure 2a for the cylindrical morphology.

On the other hand, by direct inspection of the data in Figure 2, it seems that the two NM contributions are of nearly equal intensity (proportional to the area of the corresponding loss peak component). Any reasonable decomposition by fitting to model functions (see below) leads to the same conclusion. Furthermore, the area below the whole NM loss range seems to be very close in all samples if the comparison is made by taking into account the corresponding PI fraction. This indicates that the two NM components detected in the copolymers with lamellar morphology correspond to the complete reorientation of the PI block end-to-end vector. This feature is confirmed by the data analysis presented below, and again by the evolution of the NM when crossing the ODT (see Supp. Info.).

The difference in time scales between the two NM components is apparently smaller in PI4-PDMS3.5 than in PI4-PDMS4. Indeed the slow component in PI4-PDMS3.5, although visible, is not so well-resolved in the raw data. As already mentioned, increasing the temperature results in better-resolved NM components, which in addition puts in evidence that both components present distinct temperature dependences. The data in Figure 2 suggest that the temperature dependence of the fast NM component (in particular that of PI4PDMS4) is similar to that of pure PI4. This finding indicates that the NM fast component mainly reflects the dynamics of the internal chain (Rouse) modes. As we will show below, this is confirmed by a quantitative analysis for the two investigated lamellar copolymers.

The temperature dependence of the main NM loss peak, reflecting mostly the slow component, is less pronounced in the lamellar system than in the pure PI (see below). This fact evidences the relevance, on the NM relaxation, of other microscopic mechanisms than the PI internal chain dynamics. These additional mechanisms are likely related with the dynamics of the junction point at the lamellar interface. Indeed for a polymer tethered to a fixed point in a plane the end-to-end vector reorientation, which is responsible for the dielectric NM of PI, cannot be completed since half of the vector orientations are forbidden. Moreover, the time scale of the internal chain motions of the PI-blocks is quite close to that in pure PI 
(after correcting for the differences in the segmental mobility). This observation is rather different from that found for PI-blocks of similar molecular weight in symmetric lamellae of PI-PS [15]. Unlike in the case of PI-PDMS, where PDMS has a higher mobility than PI, in the PI-PS lamellae the PI-blocks were confined between glassy domains of PS, and the NM-peak of the PI-block was located at lower frequencies than that of the corresponding homopolymer. The observed difference was in qualitative agreement with a factor 4 , which is the exact result for a chain tethered to a fixed point without further spatial constraints. Having said this, the $2 \mathrm{~d}$-diffusion of the junction point along an infinite, perfect lamellar

plane, would not be yet an efficient mechanism for completing relaxation of the end-to-end vector either. Therefore, a more complex mechanism for the motion of the junction point must be involved in the full NM relaxation of the PI-blocks in the PI-PDMS lamellae. This might be rationalized by considering that, being the junction point motion anisotropic, there is a significant displacement in the direction perpendicular to the average lamellar interface ('lamellar plane' in the following). This would be the case for lamellar phases with either highly undulated interfaces or with relatively high concentration of defective sites. We will discuss this question in Section V.

\section{DATA ANALYSIS}

\section{A. Molecular dynamics simulations}

In order to perform an analysis of the BDS data presented above, beyond a pure phenomenological description, we use information from the simulations of the bead-spring model for symmetric diblock copolymers. On the basis of the simulation results, we establish the relaxation function describing the contribution of the internal chain modes to the experimental NM relaxation. From the simulation data we have computed the Rouse modes $\mathbf{X}_{p}$ and the correlation function of the end-to-end vector $\mathbf{R}_{\mathrm{EE}}$ for the slowest block (B-beads). The Rouse modes are defined as [33]

$$
\mathbf{X}_{p}(t)=\frac{1}{N} \sum_{n=1}^{N} \cos \left(p \pi \frac{n-1 / 2}{N}\right) \mathbf{r}_{n}(t)
$$

where $\mathbf{r}_{n}$ denotes the position of the $n$th bead in the B-block, and $N$ is the total number of beads in the block ( $N=30$ for the present case). The Rouse modes given by eq. 3 are 


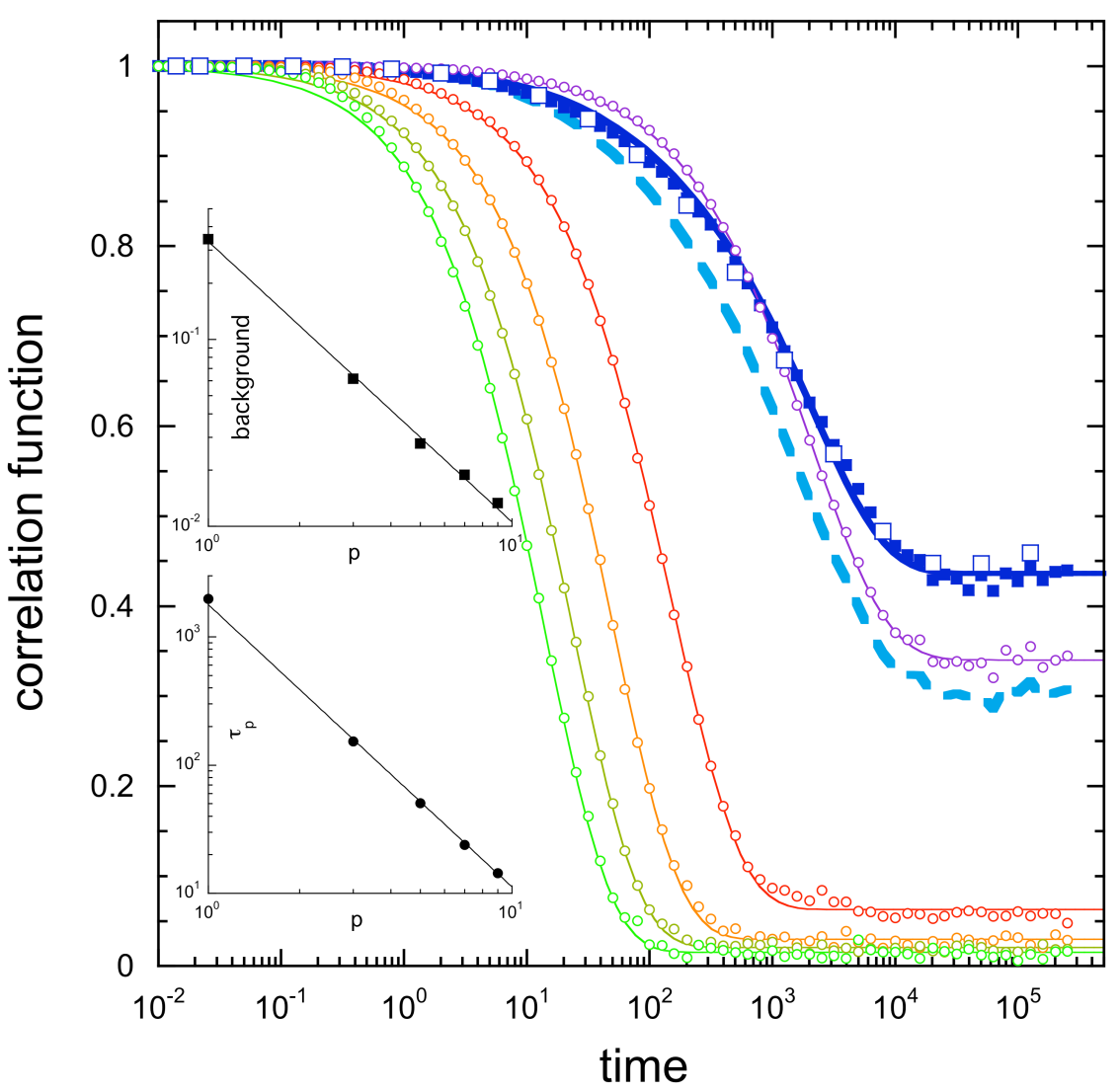

FIG. 3: Normalized correlation functions of the first odd-Rouse modes $p=1,3,5,7,9$ (small empty circles) and the end-to-end vector (small filled squares) for the slow bead-spring block, in the limit of strongly segregated lamellae $\left(\epsilon_{\mathrm{AB}}=9\right)$. For the Rouse modes, lines correspond to fits using an stretched exponential plus a background. For the end-to-end vector the solid line is obtained by using eq. 6 whereas the dashed line and the big empty squares are obtained by applying eq. 4 without and with an additional background, respectively. Insets show the $p$-dependence of the relaxation times $\tau_{p}$ and background $b_{p}$ obtained from the fitting of the block mode correlators (see text).

the exact eigenmodes of the Rouse equations of motion [33]. If the Rouse model captures the correct physics of intramolecular relaxation they will also be the actual normal modes of the chain. The Rouse modes are labelled as $p=0,1,2 \ldots N-1$, with $p=0$ being the centerof-mass position, and the other modes representing periodic deformations of wavelength $N / p$.

In Figure 3 we show the normalized correlation functions of the odd Rouse modes (up to $p=9)$ and the end-to-end vector. These quantities are respectively defined as $\varphi_{p}(t) / \varphi_{p}(0)$ 
and $\varphi_{\mathrm{EE}}(t) / \varphi_{\mathrm{EE}}(0)$, where $\varphi_{p}(t)=\left\langle\mathbf{X}_{p}(t) \cdot \mathbf{X}_{p}(0)\right\rangle$ and $\varphi_{\mathrm{EE}}(t)=\left\langle\mathbf{R}_{\mathrm{EE}}(t) \cdot \mathbf{R}_{\mathrm{EE}}(0)\right\rangle$. Data are represented for the most strongly segregated phase investigated (interaction parameter $\left.\epsilon_{\mathrm{AB}}=9\right)$. As can be seen in the figure, after a fast decay all the correlators form a plateau that persists up to the end of the simulation time window. The observed plateau is absent in the homopolymer counterpart, and constitutes a clear difference from predictions of the Rouse model. Indeed the Rouse model predicts a single exponential decay [33] for both the Rouse and end-to-end correlators. The plateau level of the Rouse correlators is strongly dependent on the mode number. Moreover, the plateau of the slowest mode correlator $(p=1)$ is larger than that of the end-to-end vector. This is also a signature of the violation of the Rouse model in the lamellar structure. According to the Rouse model, the end-to-end correlator can be exactly expressed as a sum of the correlators of the odd $(p=1,3,5 \ldots)$ Rouse modes [33]:

$$
\varphi_{\mathrm{EE}}(t)=\left\langle\mathbf{R}_{\mathrm{EE}}(t) \cdot \mathbf{R}_{\mathrm{EE}}(0)\right\rangle=16 \sum_{p \text { odd }}^{N-1}\left\langle\mathbf{X}_{p}(t) \cdot \mathbf{X}_{p}(0)\right\rangle
$$

We have computed the right-hand side of this expression by inserting there the odd Rouse correlators directly obtained from the simulations. The so-obtained theoretical end-to-end correlator is compared with the actual one in Figure 3. The comparison evidences that the actual decay to the plateau is weaker than expected from the Rouse model. Still, the time dependent parts of the theoretical and actual end-to-end correlators look rather similar. Indeed a good agreement between them is found by allowing for an additional background in Eq. 4 prior to normalization.

Guided by this result, we still try to describe the actual end-to-end dynamics, at least partially, in terms of the internal Rouse dynamics. For this purpose, we first describe each individual Rouse correlator as the sum of a time-dependent function plus a constant background. The time dependent part is fitted, in a purely empirical way, to an stretched exponential function. With this, the fitting function is given by:

$$
\varphi_{p}(t)=\left\langle X_{p}^{2}(0)\right\rangle\left[\left(1-b_{p}\right) e^{-\left(t / \tau_{p}\right)^{\beta_{p}}}+b_{p}\right]
$$

In this expression $\left\langle X_{p}^{2}(0)\right\rangle$ is the amplitude of the (non-normalized) Rouse correlator, which accounts for the intramolecular static correlations [33]. The parameters $\beta_{p}$ and $\tau_{p}$ are, respectively, the stretching exponent and the time scale of the time-dependent part. The parameter $b_{p}$ is the background of the normalized Rouse correlator. In the Rouse model, the 
correlators have no background $\left(b_{p}=0\right)$ and decay exponentially $\left(\beta_{p}=1\right)$. Moreover, both amplitudes and relaxation times scale as [35] $\left\langle X_{p}^{2}(0)\right\rangle, \tau_{p} \propto \sin ^{-2}(p \pi / 2 N)$. For simplicity, in the following we will compare simulation results with the low- $p$ approximation of the Rouse scaling, i.e, $\left\langle X_{p}^{2}(0)\right\rangle, \tau_{p} \propto(p \pi / 2 N)^{-2}$. For the block lengths of our systems this approximation is satisfactory at least for $p<6$ (error below $4 \%$ ). Since indeed this $p$-range, which has the largest amplitudes and longest relaxation times, fully dominates the slow relaxation of the end-to-end vector, the former simplification is justified.

Regarding the fits to eq. 5 , we leave $\tau_{p}, \beta_{p}$, and $b_{p}$ as fit parameters and fix $\left\langle X_{p}^{2}(0)\right\rangle$ to the values directly obtained from the simulations. For these we find $\left\langle X_{p}^{2}(0)\right\rangle \sim p^{-2.2}$. This is the same result observed in simulations of similar bead-spring models for homopolymers $[35,36]$. This result indicates that, in the simulation, the blocks in the lamellar phase esentially maintain their Gaussian character $\left(\sim p^{-2}\right)$, without significant chain stretching, as anticipated in sesction III A. Therefore the static properties of the homopolymer chains are not significably altered in the lamellar phase. Regarding the dynamics, instead of the strictly exponential $\left(\beta_{p}=1\right)$ behavior predicted by the Rouse model, we find values of $\beta_{p} \sim 0.75$ (varying monotonously from 0.7 for $p=1$ to 0.8 for $p=9$ ). Actually, deviations from pure exponential behavior have been reported even in simulations of similar bead-spring homopolymers $[35,36]$. However, in the case of homopolymers the observed stretching $\left(\beta_{p} \sim 0.85\right)$ is significantly weaker than in the lamellar systems investigated here. The resulting fits to eq. 5 are plotted in Figure 3. The inset shows the $p$-scaling obtained for both the time scale $\tau_{p}$ and the background level $b_{p}$. The relaxation times exhibit the scaling behavior $\tau_{p}=\tau_{1} p^{-2.2}$, very close to the strictly Rouse-like scaling $\left(\tau_{p}=\tau_{1} p^{-2}\right)$ observed for bead-spring homopolymers $[35,36]$. On the other hand, the background parameter $b_{p}$ scales in good approximation as $p^{-1.5}$. The former results suggest that the time-dependent part of the Rouse correlators of the blocks, prior to the plateau, retains the microscopic mechanism of the homopolymer counterpart, and therefore can be reasonably described by Rouse dynamics of the intramolecular modes. On the contrary, the plateau is a specific feature connected with the presence of the interface in the lamellar structure.

With these ideas in mind, we may still try to describe the time-dependent part of the end-to-end correlator as a sum of the odd correlators of the Rouse modes, as in eq. 4. For this purpose we fix the correlators $\varphi_{p}(t)$ to stretched exponentials. According to the former fits we now fix, for simplicity, $\beta_{p}=0.75, \tau_{p}=\tau_{1} p^{-2.2}$, and $\left\langle X_{p}^{2}(0)\right\rangle=\left\langle X_{1}^{2}(0)\right\rangle p^{-2.2}$ (see 
above). Then, we describe the normalized end-to-end correlator as,

$$
\varphi_{\mathrm{EE}}(t) / \varphi_{\mathrm{EE}}(0)=b_{\mathrm{EE}}+\left(1-b_{\mathrm{EE}}\right)\left[\sum_{p \text { :odd }}^{N-1} \frac{1}{p^{x}}\right]^{-1} \sum_{p \text { :odd }}^{N-1} \frac{\exp \left[-\left(t p^{x} / \tau_{1}\right)^{\beta}\right]}{p^{x}}
$$

where $x=2.2, \beta=0.75$, and $b_{\mathrm{EE}}$ is a free parameter accounting for the plateau of the end-to-end correlator. We find $b_{\mathrm{EE}}=0.44$. The corresponding fit is shown in Figure 3 . A satisfactory description of the simulation data is obtained. Finally, it should be noted that from the simulations we cannot address whether the correlation functions will present a slower decay at longer times. In that case the detected plateau level would correspond to the first stage of a slower component. This aspect will be discussed in detail below.

\section{B. BDS data analysis}

Having in mind the results of the analysis of the simulation data, we fit the BDS data in the NM range to a superposition of two contributions. In analogy with the simulations, the fast contribution represents the internal PI chain dynamics, and is built as a superposition of the odd Rouse modes. Following the scheme of eq. 6 in the frequency domain, the resulting fit function for the experimental NM reads

$$
\varepsilon^{\prime \prime}(\omega) / \Delta \varepsilon=B \Phi_{s}^{\prime \prime}\left(\omega ; \tau_{s}, \alpha_{s}\right)+(1-B) \Phi_{f}^{\prime \prime}\left(\omega ; \tau_{1}, \alpha_{f}\right)
$$

where $\Delta \varepsilon$ is the dielectric strength associated to the whole end-to-end reorientation of the PI-block, $\Phi_{s}^{\prime \prime}\left(\omega ; \tau_{s}, \alpha_{s}\right)$ represents the slow process and

$$
\Phi_{f}^{\prime \prime}\left(\omega ; \tau_{1}, \alpha_{f}\right)=\left(\sum_{p: \text { odd }}^{N-1} \frac{1}{p^{x}}\right)^{-1} \sum_{p: \text { odd }}^{N-1} \frac{\Phi_{p}^{\prime \prime}\left(\omega ; \tau_{p}, \alpha_{p}\right)}{p^{x}}
$$

represents the fast process, i.e., the relaxation of the internal Rouse modes. It is worth mentioning that the BDS results were obtained in samples containing lamellar domains that were randomly oriented and consequently, the detected signal arises from the end-to-end vector fluctuations projected in all directions. This justifies using an isotropic Rouse-like description for the internal chain fluctuations (i.e., the eq. 8). Moreover, broadening effects in the signal from the individual modes might originate from the random orientation of the lamellae in the BDS experiments. Having noted this, random orientation is not the only possible source of broadening in the signal. Indeed BDS experiments on PI confined in 
oriented PS-lamellae [15] revealed an extremely broad relaxation of the NM of PI, suggesting an intrinsic non-exponential character of the mode correlators. Though in the PI-PDMS lamellae investigated here the situation is somehow different - PI is confined between PDMS domains of higher mobility, unlike the glassy PS domains of Ref. [15] — the simulation resuls indicate that the Rouse modes will also have a non-exponential character. Whereas stretched exponential functions are adequate for describing an heterogeneous response in the time domain, Cole-Cole (CC) functions [14] — i.e. loss peaks with a symmetric broadening respect to the Debye ones - are often used in the frequency domain. Therefore we use CC functions, again in a purely empirical fashion, to account for the non-exponential character of both $\Phi_{s}^{\prime \prime}$ and $\Phi_{p}^{\prime \prime}$. For the sake of clarity, in eqs. 7 and 8 we indicate explicitly the two parameters of the CC functions. These are the shape parameters $\left(\alpha_{s}\right.$ and $\left.\alpha_{p}\right)$ and the peak time scales $\left(\tau_{s}\right.$ and $\left.\tau_{p}\right)$ of the $\mathrm{CC}$ functions. It must be stressed that, according to the analysis of the simulation results, we have used the same shape parameter $\alpha_{p}=\alpha_{f}$ for all the Rouse $p$-modes. Since moreover all the times $\tau_{p}$ are proportional to $\tau_{1}$ (see below), the fast component $\Phi_{f}^{\prime \prime}$ actually depends on just two parameters $\left(\tau_{1}\right.$ and $\left.\alpha_{f}\right)$. In a recent work by some of us [37] the NM of pure, non-entangled PI, was analyzed in terms of eqs. 7 and 8 by using $B=0$ (i.e., single NM peak resulting from chain dynamics), and the strict Rouse-scaling for the times and static amplitudes, $\tau_{p},\left\langle X_{p}^{2}(0)\right\rangle \sim p^{-2}$. As aforementioned, slight differences are found for the blocks in the simulations $\left(\sim p^{-2.2}\right)$. In the analysis of the BDS data for the lamellae, we have found no significant differences within statistics by using the scaling $\sim p^{-2.2}$, or by keeping that of pure PI. Fits presented in the following will correspond to this second choice $(x=2$ in eq. 8$)$.

Having said this, we needed to fix $B=0.5$ for the weigth factor in eq. 7. Attempts to use significantly smaller values of $B$, (i.e, consistently with the plateau of the simulation end-to-end correlator, see Figure 3) failed to give reasonable fits of the experimental data. As it will be discussed below, this has important consequences for the interpretation of the experiments. Taking all these points into account, a total of five fit parameters are used to describe the whole dielectric NM relaxation of the PI block in the lamellae. These are the NM relaxation strength, and the two time scales and the two shape parameters of the functions $\Phi_{p}^{\prime \prime}$ and $\Phi_{s}^{\prime \prime}$. An additional function $\Phi_{\alpha}^{\prime \prime}$ is necessary to account for the overlapping contributions in the high frequency side of the NM loss peak, which originate from the low frequency side of the PI segmental dynamics. Again a CC relaxation function is used for 
this contribution — note that this is not adequate for describing the whole $\alpha$-process [14], but it is sufficient for the present purpose.

As mentioned earlier (see also Figure 2), in the window $T<250 \mathrm{~K}$ the position of the $\alpha$-peak of the PI-block follows the same behavior as pure PI, but with a temperature shift of nearly $5 \mathrm{~K}$. Therefore, at higher temperatures we fix the $\alpha$-peak of the PI-block according to the $T$-dependence of the $\alpha$-peak of the pure PI already reported in the literature [37], and taking into account the mentioned shift. The CC shape parameter $\left(\alpha_{\alpha}\right)$ obtained for the function $\Phi_{\alpha}^{\prime \prime}$, describing the part of the $\alpha$-relaxation overlapping with the NM, is found to increase smoothly from 0.45 to 0.55 up to $T=250 \mathrm{~K}$. A value of $\alpha_{\alpha}=0.55$ has been imposed at higher temperatures. After this, for temperatures above $250 \mathrm{~K}$ the amplitude of $\Phi_{\alpha}^{\prime \prime}$ is the only additional fitting parameter to those defined above. Representative examples of the fits, below and above $250 \mathrm{~K}$, are shown in Figure 2 as lines. A satisfactory description of the experimental data is obtained over the whole investigated temperature range. Moreover, the dielectric strength, $\Delta \varepsilon$, of the whole range of the NM in the PI-phases is consistent with the expected value (see last column in Table I). This confirms that the fitting procedure accounts for the whole end-to-end reorientation of the PI-blocks.

\section{DISCUSSION}

The main fit parameters in the analysis of the BDS data for the NM are the characteristic relaxation times, $\tau_{s}$ for the slow process, and $\tau_{1}$ for the fast one resulting from the PI-block Rouse modes (note again that the times for the other modes are just obtained as $\tau_{p}=\tau_{1} p^{-2}$ ). The times $\tau_{s}$ and $\tau_{1}$ are plotted vs. temperature in Figure 4, together with the obtained values for the full-width at half-maximum $(F W H M)$ derived from the shape parameters of the respective $\mathrm{CC}$ functions as

$$
F W H M=-0.477+1.621 \alpha^{-1}
$$

(see Ref. [38]), with $\alpha=\alpha_{s}$ for the slow NM-component and $\alpha=\alpha_{f}$ for the first Rouse mode. For comparison, we also include the results for $\tau_{1}$ of the pure PI, once shifted by $5 \mathrm{~K}$ according to the difference in the respective segmental relaxation times (see above). As anticipated in Figure 2, the slowest internal chain mode $(p=1)$, which dominates the contributions to the fast NM-component in the lamellar PI-phase (note the weighting by $p^{-2}$ 
of $\Phi_{p}^{\prime \prime}$ in eq. 8) is close to, but slightly faster than that of the pure PI when the comparison is made for temperatures where the PI-blocks and the pure PI show similar segmental relaxation times. The differences are however more evident for the lamellar phase with a higher content of PDMS. This suggests that the fast local motions of the copolymer junction point, in contact with a highly mobile PDMS phase, might be at the origin of this acceleration of the chain dynamics. Concerning the slow NM-component, its temperature dependence is different (less steep) from that of the block internal chain dynamics. Interestingly, the time scale of this component depends dramatically on the PDMS-block size, being much slower for the copolymer with the somewhat larger PDMS block. However, the two time scales (slow component and internal modes) in both copolymers tend to merge at the lowest accessible temperatures, where the two different NM-components can hardly be resolved in the experiments.

As discussed above, the origin of the slow NM-component might be related with the motion of the junction point in the transverse direction, since a purely $2 \mathrm{~d}$-diffusion along the lamellar plane cannot lead to full reorientation of the end-to-end vector. For this transverse motion there are at least three (non-excluding) possible mechanisms one may consider: i) diffusion of the whole PI block through the PDMS-domain, leading to chain flip. ii) diffusion of the junction point along a highly defective/curved lamellar interface, which might be expected for diblock copolymers with relatively small block sizes (see below); iii) the presence of highly mobile grain boundaries and/or defects that would locally distort the lamellar structure. In order to address this point, we have analyzed the results for the end-to-end correlators obtained in a series of simulations with different values of the crossinteraction parameter $\epsilon_{\mathrm{AB}}$, covering the range from fully isotropic to strongly segregated phases. In the limit of strongly segregated lamellae $\left(\epsilon_{\mathrm{AB}}=9\right)$, the relaxation of the endto-end vector correlator is not completed within the simulation time window (see Figure 5). Instead, the correlator decays to a plateau value of about 0.43 , somewhat lower than that expected for a polymer chain tethered to a plane (0.5). The plateau persists until the end of the simulation. Figure 5 also shows the effect of the interaction parameter $\epsilon_{\mathrm{AB}}$ on the relaxation of the end-to-end vector. By decreasing $\epsilon_{\mathrm{AB}}$ the first-step decay becomes more pronounced, leading to a lower plateau. For example, the plateau is about 0.33 for $\epsilon_{\mathrm{AB}}=1.5$. This decrease of the plateau is accompanied by a clear signature of the beginning of a second decay. This is confirmed at lower values of $\epsilon_{\mathrm{AB}}$, for which the plateau height 


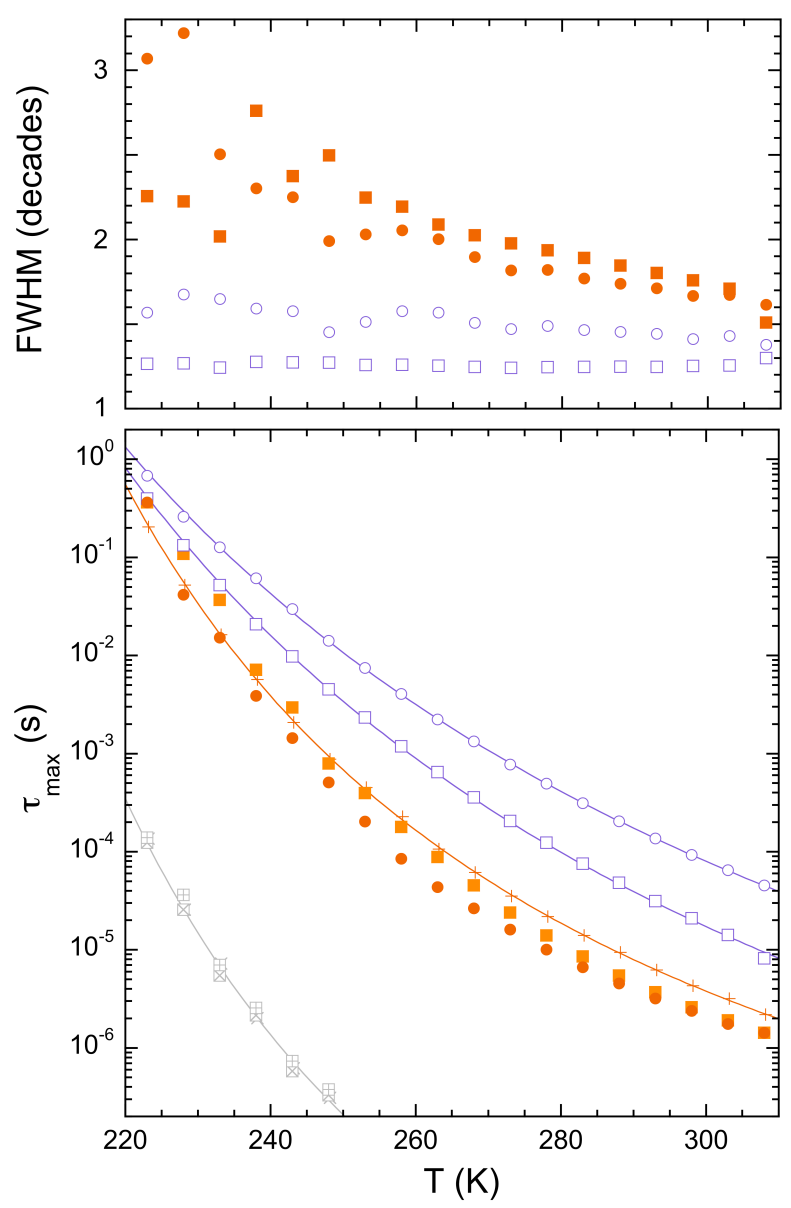

FIG. 4: Top panel: $T$-dependence of the $F W H M$ for the slow NM-component and the first Rouse mode of the PI-blocks, in the two investigated copolymers. The values of the $F W H M$ are obtained through eq. 9 from the respective shape parameters $\left(\alpha_{s}\right.$ and $\alpha_{f}$ for the slow NM-component and first Rouse mode, respectively). Bottom panel: For the PI-blocks, $T$-dependence of the relaxation times of the slow $\left(\tau_{s}\right)$ and fast $\left(\tau_{1}\right)$ NM components, as well as of the $\alpha$-relaxation times $\left(\tau_{\alpha}\right)$. For comparison we include data of $\tau_{\alpha}$ and of $\tau_{1}$ (single NM) for pure PI (with a temperature shift of $5 \mathrm{~K}$ respect to data for the PI-blocks, see above). Symbol codes in both panels for the different investigated systems are as follows. Squares: PI-blocks in PI4-PDMS3.5; circles: PI-blocks in PI4-PDMS4; crosses: pure PI. Symbol colors for the different dynamic processes are as follows. Blue: $\tau_{s}$ and $\alpha_{s}$; orange: $\tau_{1}$ and $\alpha_{f}$; grey: $\tau_{\alpha}$.

tends to vanish. Thus, for $\epsilon_{\mathrm{AB}}=0.85$ the first decay accounts for more than the $80 \%$ of the whole decay, and in the second step the relaxation of the end-to-end vector is completed within the simulation time scale. For even lower values of $\epsilon_{\mathrm{AB}}$ the plateau tends to vanish and the relaxation of the block end-to-end vector seems to converge to a single-step decay, 
as in the case of homopolymers.

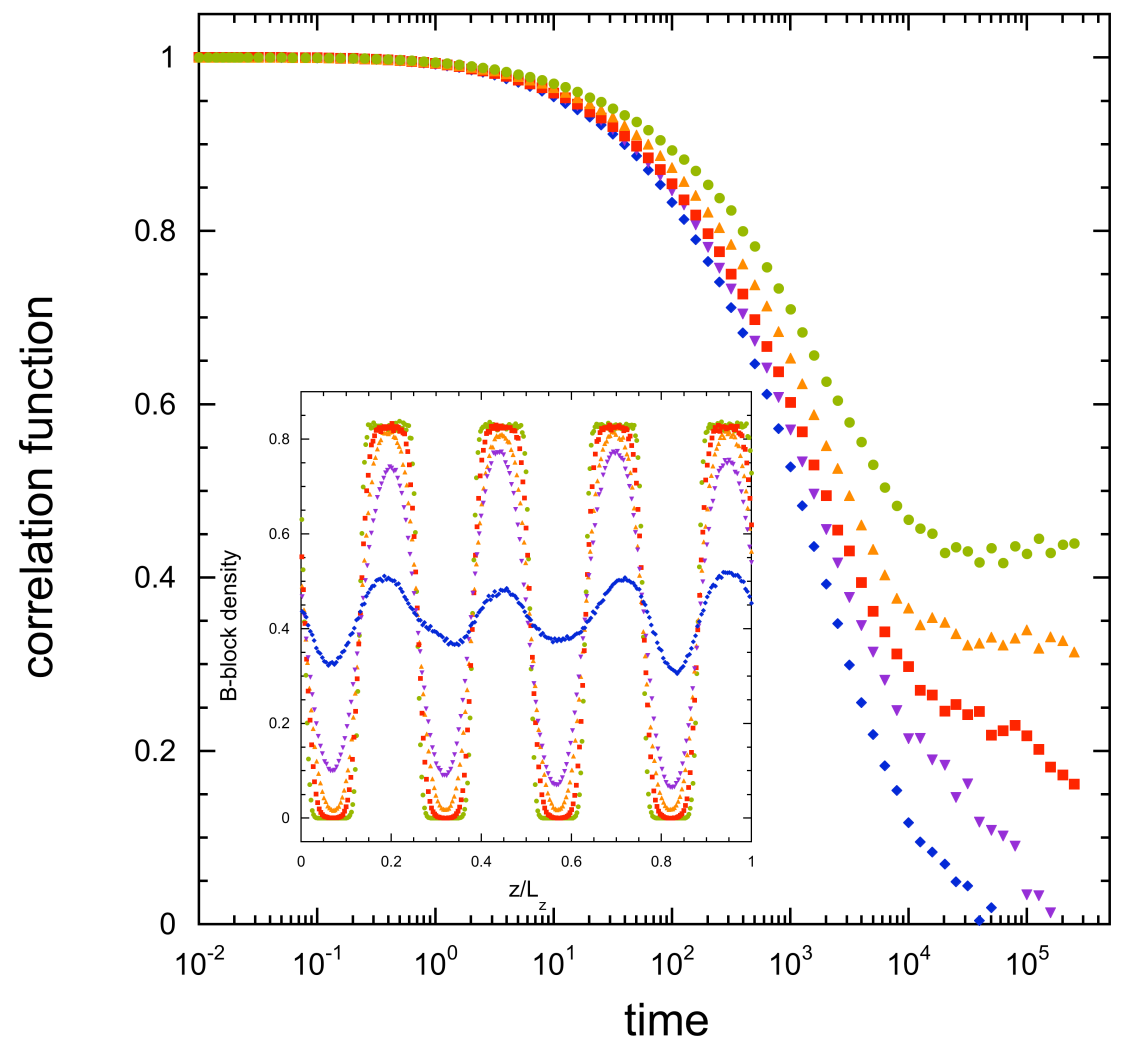

FIG. 5: End-to-end vector correlation functions of the slow B-block, for several values of the interaction parameter: $\epsilon_{\mathrm{AB}}=9$ (circles), 1.5 (triangles), 1.0 (squares), 0.85 (inverse triangles), and 0.80 (diamonds). The inset shows, with the same symbol codes, the corresponding density profiles in the transverse direction.

The inset of Figure 5 displays the density profiles of B-monomers along the $z$-direction (perpendicular to the lamellar planes), as a function of the interaction parameter $\epsilon_{\mathrm{AB}}$. This representation demonstrates that the former changes in the end-to-end correlator are intimately connected with the lower degree of segregation produced by decreasing $\epsilon_{\mathrm{AB}}$. A weaker segregation and consequently a broader, strongly fluctuating interface, leads to a significant displacement of the junction point in the z-direction. This is further demonstrated in Figure 6 , which shows simulation values of the mean-squared displacement of the junction point for different interaction parameters. Both the normal $\left(\left\langle\Delta r_{z}^{2}(t)\right\rangle\right)$ and the parallel $\left(\left\langle\Delta r_{\|}^{2}(t)\right\rangle\right)$ displacements have been calculated for each case, although not all the parallel displacements are included in the figure for the sake of clarity. From this figure, it is clear that the parallel 
component of the junction point displacement is nearly independent on the degree of segregation. However, when the transverse displacement is considered, it is evident that this is rather limited for strongly segregated lamellae. As can bee seen in Figure 6 , for $\epsilon_{\mathrm{AB}}=1.5$ and 9 the transverse displacement saturates to a few bead diameters, forming a plateau that persists until the end of the simulation. On the contrary, in the systems with weaker segregation the anisotropy in the displacement of the junction point diminishes and eventually dissapears. This feature is concomitant with the progressive disappearance of the plateau and the full relaxation observed in the end-to-end correlator (Figure 5). In summary, from the analysis of the simulations we conclude that the appearance of a second relaxation step in the end-to-end vector correlation function is directly connected with the distortion of the lamellar structure. This distortion is accompanied by an increasing relevance of the internal chain modes in the relaxation of the end-to-end vector - since these are responsible for the first decay, which becomes more pronounced for weaker segregation.

When compared with the experiments, this represents a major difference. As mentioned earlier, in the experiments the internal chain modes can account only for approximately $50 \%$ of the NM relaxation over the whole temperature range investigated. The strength of the NM relaxation mediated by the Rouse modes is overestimated by the simulated model, even for the most strongly segregated case $\left(\epsilon_{\mathrm{AB}}=9\right.$, with a plateau of $\approx 0.43$ in the normalized end-to-end correlator, see Figure 5). Moreover, the experimental slow-NM component presents time scales of less than 2 decades longer than the fast component. In the simulations, for $\epsilon_{\mathrm{AB}}=9$ a much larger separation is expected between the first decay and the second one (the latter eventually occurring far beyond the simulation window). Furthermore, in the experiments the time scale difference between the fast and the slow NM components increases with temperature (see Figure 4), whereas the segregation quality would eventually decrease. Instead, in the simulations decreasing lamellar quality (by lowering $\epsilon_{\mathrm{AB}}$ ) leads to a smaller separation between the two processes (Figure 5). According to all these results, the experimentally observed second step-decay of the end-to-end vector correlation function cannot be connected to that observed in the simulations by approaching the ODT. Indeed, data presented in Figure 2 are not close to the ODT but correspond to states in strongly segregated lamella, as confirmed by the analysis of the SAXS data (e.g., by the law $Q_{\max } \sim \chi^{-1 / 6} N^{-2 / 3}$, see Figure 1). Only experiments around the ODT could be directly compared with the simulations. 


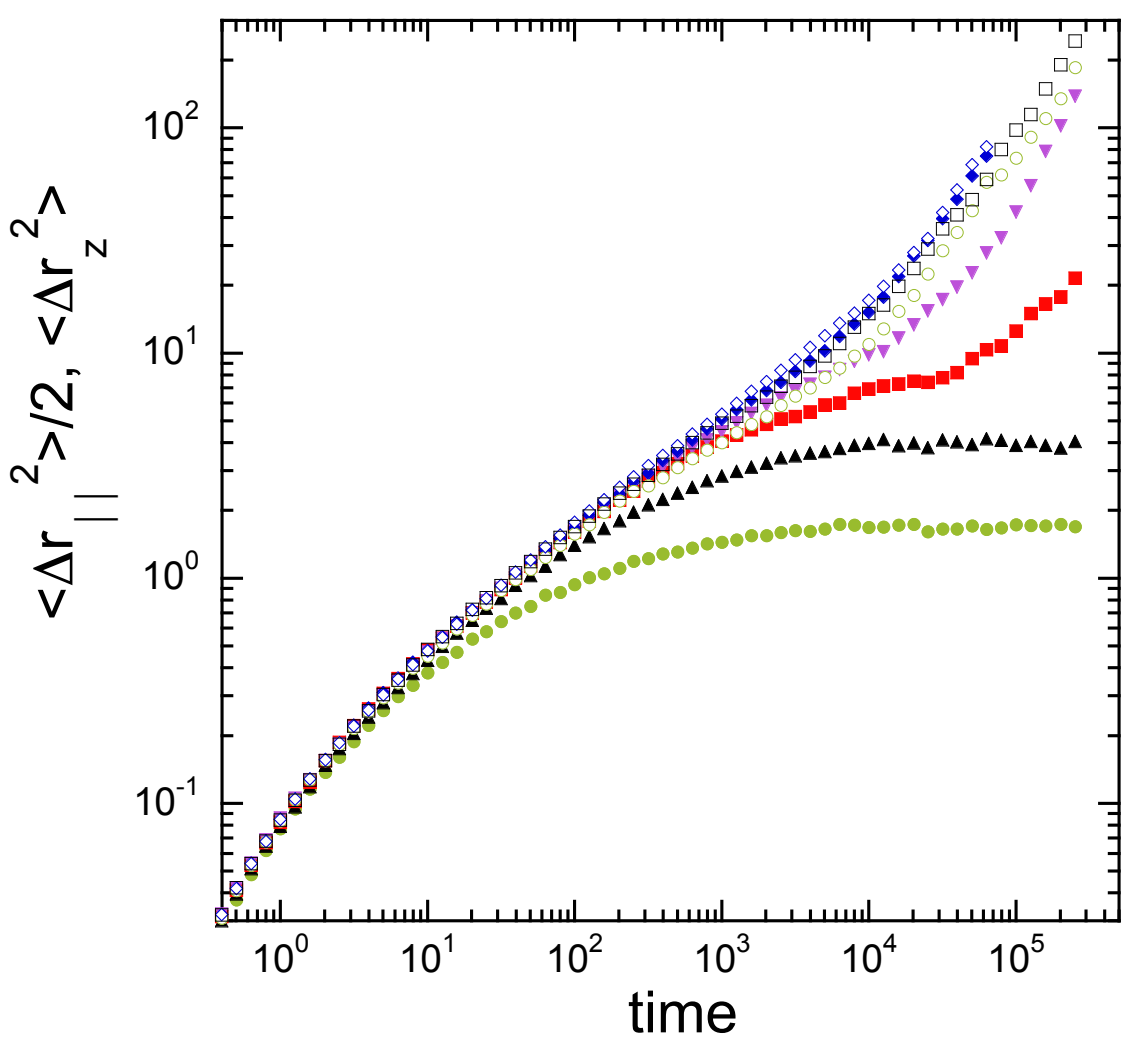

FIG. 6: Simulation results for the mean squared displacements of the junction point in the parallel (empty symbols) and perpendicular (filled symbols) directions, for diblocks of varying interaction parameters. Symbol codes are as in Figure 5.

A possible origin of the differences between experiments and simulations, with respect to the slow component of the end-to-end relaxation, might be the presence of structural features in the real system that favour transverse motions of the junction point but are not captured by the simulation model. An experimental feature that might shed light on the origin of the NM slow component is the rather different temperature dependence of this component in comparison with that of the PI chain dynamics. A quantitative description of the temperature dependence of the characteristic relaxation times can be obtained by using the Vogel-Fulcher-Tamman (VFT) equation [39],

$$
\tau(T)=\tau_{\infty} \exp \left[\frac{B}{T-T_{0}}\right]
$$

where $T_{0}$ is the so-called Vogel temperature, $B$ is an energetic term, and $\tau_{\infty}$ provides the high-temperature limit of the relaxation time. The lines in Figure 4 correspond to VFT-fits 


\begin{tabular}{cccc}
\hline & $T_{0}(K)$ & $B(K)$ & $\tau_{\infty}(s)$ \\
\hline & & & \\
PI4-PDMS3.5 & 110.0 & 2816 & $6.3 \times 10^{-12}$ \\
PI4-PDMS4 & 95.2 & 3109 & $2.1 \times 10^{-11}$ \\
PI4 & 150.4 & 1549 & $1.2 \times 10^{-10}$
\end{tabular}

TABLE II: VFT parameters of the characteristic relaxation times of the slow NM-component of the PI-blocks in the two investigated lamellar systems. For comparison we include the parameters of the NM of pure PI.

of the experimental times.

The resulting VFT-parameters for the times of the slow NM-component, $\tau_{s}$, of the PIblocks are shown in Table II. For comparison, we include the results for the single NM time, $\tau_{1}$, of pure PI. The values for $B$ and $T_{0}$ corresponding to the slow NM-component are markedly different from those of the NM of pure PI. In particular $T_{0}$ in the lamellar phase is about $50 \mathrm{~K}$ lower, whereas $B$-values are nearly twice that of the NM of pure PI. Thus, it is evident that the mechanism responsible for the slow NM component in the lamellar diblocks must be highly affected by the presence of the highly mobile PDMS phase. Given the short time scale separation between the two components of the NM, we discard interdomain diffusion as a relevant mechanism for the present case. Note that in the simulations this would be the only possible mechanism for long transverse motions in the limit of strong segregation (e.g., $\epsilon_{\mathrm{AB}}=9$ ), and simulation data reveal a much larger time scale separation between the first and the (eventual) second decay.

It must be noted that, though in the limit of strong segregation stiff and narrow interfaces are obtained, multi-domain lamellar arrangements are expected at large scales for systems of short chains as those investigated here. These arrangements involve large wavelength interface undulations, grain boundaries or other defects, which are not captured by the limited size of the simulation box. Moreover, it must be noted that the simulated system is prepared and equilibrated in a perfect lamellar geometry (see protocol in Ref. [18]). In a recent theoretical work [40] a mechanism based on the diffusion of defects in multi-domain lamellar configurations was found to allow for relaxation in lamellar phases. Moreover, the relaxation rate originated by grain boundary motions was found to be faster than that of 
the whole diblock diffusion. It must be noted that the whole diblock diffusion is hindered by the block of lower intrinsic mobility (PI in the present case), and therefore its temperature dependence would be expected to be similar to that of the normal mode of pure PI. Furthermore, no large changes in the block diffusion should be expected by a rather minor variation in the size of the PDMS block, as in the case of the investigated systems PI4-PDMS4 and PI4-PDMS3.5. Therefore, according to the above discussion the origin of the slow NMcomponent might be the presence of junction point motions of relatively large amplitude, facilitated by relatively fast moving defects, grain boundaries or interfacial undulations. Such defect-based mechanisms might be also present in phases with curved interphases, as the cylindrical, spherical and gyroid morphologies. However, in these phases, unlike in the lamellar case, the diffusion of the junction point along the interface is sufficient by itself for completing the NM relaxation, irrespectively of the presence of defect-based mechanisms. The absence of bimodality for the NM of the cylindrical system of Figure 2 suggests that the internal chain mode dynamics and the motion of the junction point have similar time scales. Bimodality of the NM for curved domains should arise when there is a significant time scale separation between both processes. This might be the case, e.g., when the block is coupled to an slower component, leading to an slowing down of the diffusion along the interface. Still, unlike in the lamellar case, this diffusion would be sufficient to complete the NM relaxation even without intervening defect-based mechanisms.

As discussed above, the mentioned mechanisms for junction point motion in the lamellar phase would be consistent with the relatively weak temperature dependence of the NMslow component, and also with the strong impact produced by a relatively minor change in the size of the PDMS block. In relation with the latter, a close inspection of the SAXS patterns for both copolymers evidences that the main diffraction peak is sharper for PI4PDMS4 than for PI4-PDMS3.5, particularly in the bottom part. This suggests a better quality of the lamellar structure in PI4-PDMS4. In order to quantify the quality of the lamellae, we describe the main diffraction peak as the superposition of a Gaussian and a Lorentzian component (see lines in Figure 1a). We find that in PI4-PDMS4 the Lorentzian component accounts only for $15 \%$ of the total intensity. However in PI4-PDMS3.5 a much higher contribution (29\%) is observed, reflecting a significantly lower quality of the lamellar structure. Thus, the lower amount of defects in the PI4-PDMS4 copolymer would rationalize why a longer time is required for the complete end-to-end vector reorientation in this system 
as compared to PI4-PDMS3.5.

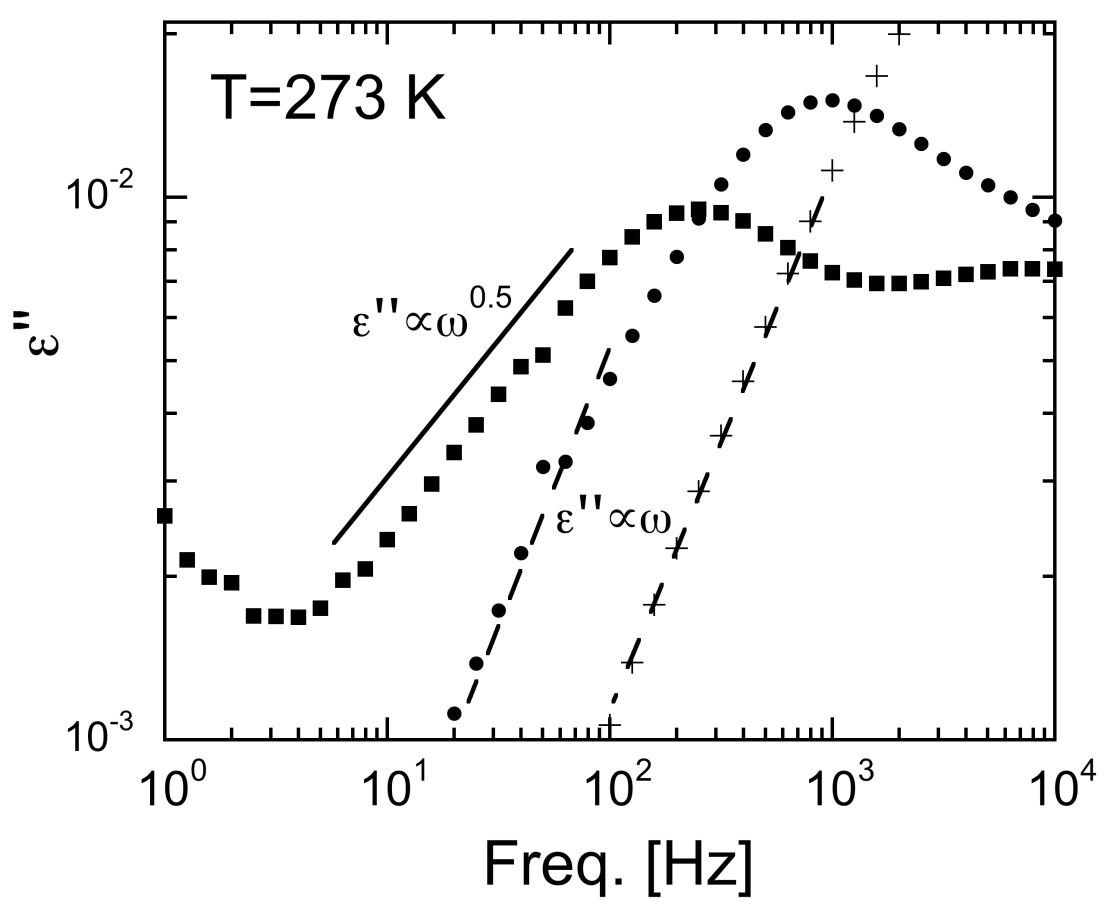

FIG. 7: Low-frequency behavior, at $273 \mathrm{~K}$, of the dielectric relaxation of the PI-blocks in PI4-PDMS3.5 (circles) and PI4-PDMS4 (squares), as compared with that of pure PI4 (crosses). Dashed lines represent the linear behavior, $\epsilon^{\prime \prime} \propto \omega$, expected for the terminal relaxation of homopolymers. The solid line corresponds to the anomalous result $\epsilon^{\prime \prime} \propto \omega^{0.5}$ (see text).

Another observation that may support this interpretation is the low-frequency behavior of the slow component of the NM. Figure 7 shows the BDS results for the two copolymers and PI-homopolymer in double logarithmic scale, in order to visualize features in the lowfrequency regime. The pure PI shows the usual linear scaling, $\varepsilon^{\prime \prime} \sim \omega$, expected for the terminal relaxation of homopolymers. Though the conductivity contribution contamites the signal of PI4-PDMS4 for $\omega<10$, anomalous behavior is unambiguosly found in the range $10 \lesssim \omega \lesssim 100$. This can be effectively described by a power-law $\varepsilon^{\prime \prime} \sim \omega^{0.5}$. This feature might be intimately related to the anomalous low-frequency behavior, $G^{\prime}, G^{\prime \prime} \sim \omega^{0.5}$, observed in the moduli for the viscoelastic response of strongly segregated lamellae [41-44]. It has been proposed that defect-based mechanisms are also responsible for this anomalous viscoelastic response [45], on the basis of a large time scale separation between block diffusion along the domains and relaxation of defects. This separation seems to be much weaker in the case 
of PI4-PDMS3.5. Thus, the corresponding BDS data do not exhibit the anomalous scaling $\varepsilon^{\prime \prime} \sim \omega^{0.5}$ observed for PI4-PDMS4. They instead follow, within statistics, linear behavior as in the pure PI.

The width of the NM-components is the other relevant parameter from the BDS analysis, see Figure $4 \mathrm{a}$. In the case of the internal chain dynamics, the $F W H M$ refers to the non-exponential character attributed to the individual Rouse modes (the $F W H M$ value corresponding to an exponential relaxation function is 1.14 decades). The results show that there is a clear trend to a more exponential character of the mode relaxation as temperature increases, both copolymers approaching similar values within fitting uncertainties. Still, the Rouse modes of the PI4-PDMS3.5 copolymer seem to exhibit a systematically broader relaxation. Again, this might be justified by the lower quality of the lamellar structure in that system, leading to strong dynamic heterogeneity. In the high temperature range, the $F W H M$ values are still distinguishably above that of pure PI ( $F W H M=1.21$ decades). This is consistent with the more stretched character of the non-exponential decay of the Rouse modes in the simulations of the copolymers, as compared to those of the bead-spring homopolymer (see above). On the other hand, the width of the experimental slow NMcomponent is in both copolymers rather small ( $F W H M<1.7$ decades) as compared to that of the fast NM-component. This suggests a well-defined time scale and therefore, in the proposed framework, a well-defined defect diffusion coefficient. However, the width of the slow NM-component is significantly larger for PI4-PDMS4. This is directly connected to the anomalous low-frequency scaling $\varepsilon^{\prime \prime} \sim \omega^{0.5}$ discussed above.

\section{CONCLUSIONS}

By using dielectric spectroscopy, we have investigated the chain dynamics of nonentangled PI under soft confinement in lamellar domains of block copolymer melts with PDMS. The data reveal two distinct mechanisms relevant for the NM relaxation. The fast component can be attributed to the relaxation of the internal chain modes, which has been rationalized according to the analysis of the Rouse modes in a generic bead-spring model for strongly segregated diblock copolymers. The analysis of the NM of the PI-blocks in the copolymers shows that the internal chain modes have relaxation time scales close to those found in pure PI, once the temperature shift induced by the PDMS phase on the PI segmen- 
tal relaxation is considered. On the contrary, the slow NM-component, which is attributed to the dynamics of the junction point, shows a time scale with a markedly different temperature dependence as compared with the PI chain dynamics. Moreover, this time scale depends critically on the minor structural differences between the two investigated lamellar systems. The diblock bead-spring model cannot reproduce this effect, indicating that in the real systems the slow NM-component is affected by structural characteristics that are not captured by the limitted size of the simulation box. The former features for the slow NM-component might be tentatively explained as originating from the presence of relatively fast moving defects and/or grain boundaries in the lamellae. The presence of fast moving defects would be responsible for large excursions of the junction point in the direction perpendicular to the lamellar interface, which are required in order to relax completely the end-to-end vector autocorrelation function.

\section{Acknowledgements}

We acknowledge the financial support from Projects No. MAT2012-31088 (Spanish Government) and No. IT654-13 (Basque Government). The X-ray measurements were performed with the SAXS instrument at the Centro de Física de Materiales (CFM) (CSIC-UPV/EHU) acquired with the nominative subvention of the MEC to the CFM 2008.

[1] Hamley, I.W. The Physics of Block Copolymers, Oxford University Press, Oxford, UK, 1998.

[2] Bates, F.S.; Fredrickson, G.H. Ann. Rev. Phys. Chem., 1990, 41, 525.

[3] Park, S.; Lee, D.H.; Xu, J.; Kim, B.; Hong, S.W.; Jeong, U.; Xu, T.; Russell, T.P. Science, 2009, 323, 1030.

[4] Segalman, R. A.; Yokoyama, H.; Kramer, E. J. Adv. Mater., 2001, 13, 1152.

[5] Leibler, L. Macromolecules, 1980, 13, 1602.

[6] Fredrickson, G.H.; Helfand, E. J. Chem. Phys., 1987, 87, 697.

[7] Fredrickson, G.H.; Helfand, E. J. Chem. Phys., 1987, 89, 598.

[8] Fredrickson, G.H.; Bates, F.S. Ann. Rev. Mater. Sci., 1996, 26, 501.

[9] Dalvi, M.C.; Eastman, C.E.; Lodge, T.P. Phys. Rev. Lett., 1993, 71, 2591. 
[10] Lodge, T.P.; Dalvi, M.C. Phys. Rev. Lett., 1995, 75, 657.

[11] Hamersky, M.W.; Hillmyer, M.A.; Tirell, M.; Bates, F.S.; Lodge, T.P.; von Meerwall, E.D. Macromolecules, 1998, 32, 5872.

[12] Anastasiadis, S.H. Curr. Opin. Coll. Int Sci., 2000, 5, 324.

[13] Papadakis, C.M.; Rittig, F. J. Phys.: Condens. Mat., 2005, 17, R551.

[14] Kremer, F.; Schönhals, A. (eds.) Broadband Dielectric Spectroscopy, Springer-Verlag, Berlin, 2003.

[15] Yao, M.-L.; Watanabe, H.; Adachi, K.; Kotaka, T. Macromolecules, 1991, 24, 2955.

[16] Sanz, A.; Nogales, A.; Ezquerra, T.A. Soft Matter, 2011, 7, 6477.

[17] Valle-Carrandi, L.; Alegría, A.; Arbe, A.; Colmenero, J. Macromolecules, 2012, 45, 491.

[18] Slimani, M.Z.; Moreno, A.J.; Colmenero, J. Macromolecules, 2011, 44, 6952.

[19] Slimani, M.Z.; Moreno, A.J.; Colmenero, J. Macromolecules, 2012, 45, 8841.

[20] Alig, I.; Kremer, F.; Fytas, G.; Roovers, J. Macromolecules, 1992, 25, 5277.

[21] Chen, Q.; Matsumiya, Y.; Iwamoto, T.; Nishida, K.; Kanaya, T.; Watanabe, H.; Takano, A.; Matsuoka, K.; Matsushita, Y. Macromolecules, 2012, 45, 2809.

[22] Matsumiya, Y.; Chen, Q.; Uno, A.; Watanabe, H.; Takano, A.; Matsuoka, K.; Matsushita, Y. Macromolecules, 2012, 45, 7050 .

[23] Meins, T.; Dingenouts, N.; Kubel, J.; Wilhelm, M. Macromolecules, 2012, 45, 7206.

[24] R. Lund, R.; Willner, L.; Alegría, A.; Colmenero, J.; Richter, D. Macromolecules, 2008, 41, 511.

[25] Willner, L.; Lund, R.; Monkenbusch, M.; Holderer, O.; Colmenero, J.; Richter, D. Soft Matter, 2010, 6, 1559 .

[26] Montes, H.; Monkenbusch, M.; Willner, L.; Rathgeber, S.; Richter, D.; Fetters, L.J.; Farago, B. Europhys. Lett., 2002, 58, 398.

[27] Fetters, L.J.; Lohse, D.J.; Colby, R.H. Physical Properties of Polymers Handbook, ed. Mark, J.E., Springer, 2007.

[28] Kremer, K.; Grest, G. S. J. Chem. Phys., 1990, 92, 5057.

[29] This is different from the experimental approach, in which the order-disorder transition is driven by varying temperature. Since temperature variation leads to changes in relaxation times by decades, and the time scales accessed by simulations are limited, we have decided to tune the degree of structural order by varying the cross-interaction at fixed temperature. 
[30] Cochran, E. W.; Morse, D. C.; Bates, F. S. Macromolecules, 2003, 36, 782

[31] Semenov, A. N. Sov. Phys. JETP, 1985, 61, 733.

[32] Pispas, S.; Hadjichristidis, N.; Floudas, G. Block Copolymers: Synthetic Strategies, Physical Properties, and Applications, John Wiley and Sons, Inc., New Jersey, 2003.

[33] Doi, M.; Edwards, S.F. The Theory of Polymer Dynamics; Oxford University Press: Oxford, U.K., 1986.

[34] Barroso-Bujans, F; Lund, R.; Willner, L.; Richter, D.; Alegría, A.; Colmenero, J., in preparation.

[35] Bennemann, C.; Baschnagel, J.; Paul W.; Binder, K. Comput.Theor. Polym. Sci., 1999, 9, 217.

[36] Moreno, A.J.; Colmenero, J. Phys. Rev. Lett., 2008, 100, 126001.

[37] Riedel, C.; Alegría, A.; Tordjeman, P.; Colmenero, J. Macromolecules, 2009, 42, 8492.

[38] Schwartz, G.A.; Colmenero, J.; Alegría, A. Macromolecules, 2007, 40, 3246.

[39] Vogel, H. Phys. Z., 1921, 22, 645.

[40] Yoo, C.-D.; Viñals, J. Macromolecules, 2012, 45, 4848.

[41] Rosedale, J.H.; Bates, F.S. Macromolecules, 1990, 23, 2329.

[42] Koppi, K.A.; Tirrell, M.; Bates, F.S.; Almdal, K.; Colby, R.H. J. Phys. II (Fr.), 1992, 2, 1941.

[43] Larson, R.G.; Winey, K.I.; Patel, S.S.; Watanabe, H.; Bruinsma, R. Rheol. Acta, 1993, 32, 245 .

[44] Balsara, N.P., Dai, H.J.; Watanabe, H.; Sato, T., Osaki, K. Macromolecules, 1996, 29, 3507.

[45] Rubinstein, M.; Obukhov, S.P. Macromolecules, 1993, 26, 1740. 


\section{TABLE OF CONTENTS GRAPHIC}
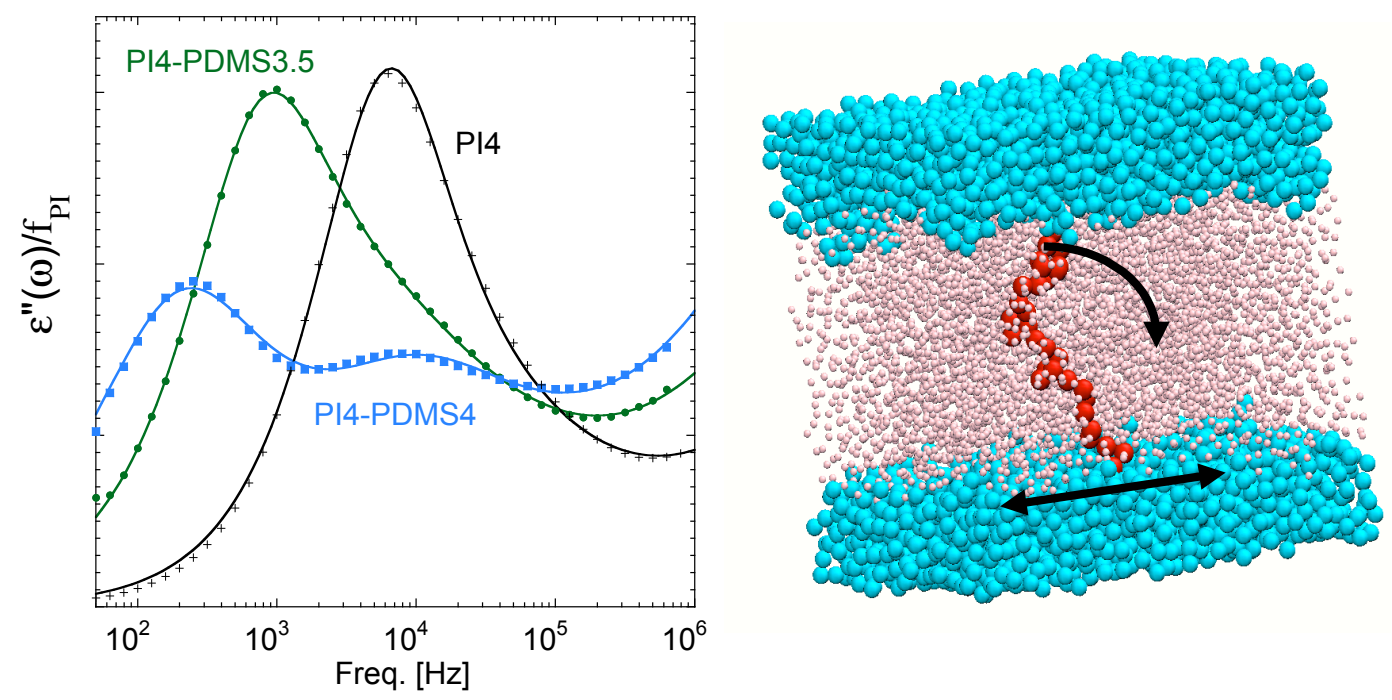

End-to-end Vector Dynamics of Non-entangled Polymers in Lamellar Block Copolymer Melts: the Role of Junction Point Motion

Reidar Lund, Fabienne Barroso-Bujans, Mohammed Zakaria Slimani, Angel J. Moreno, Lutz Willner, Dieter Richter, Angel Alegría, and Juan Colmenero 